\title{
Long non-coding RNA HOTAIR, a driver of malignancy, predicts negative prognosis and exhibits oncogenic activity in oesophageal squamous cell carcinoma
}

\author{
$\mathrm{XLi}^{1}, \mathrm{Z} \mathrm{Wu}{ }^{1}, \mathrm{Q} \mathrm{Mei}^{1}, \mathrm{X} \mathrm{Li}^{1}, \mathrm{M} \mathrm{Guo}{ }^{2}, \mathrm{X} \mathrm{Fu}^{1,3}$ and $\mathrm{W} \mathrm{Han}^{*}, 1$ \\ ${ }^{1}$ Department of Molecular Biology, Institute of Basic Medicine, School of Life Sciences, Chinese PLA General Hospital, Beijing \\ 100853, China; ${ }^{2}$ Department of Gastroenterology and Hepatology, Chinese PLA General Hospital, Beijing 100853, China and \\ ${ }^{3}$ Key Laboratory of Wound Healing and Cell Biology, Institute of Burns, the First Affiliated Hospital to the Chinese PLA General \\ Hospital, Trauma Center of Postgraduate Medical School, Beijing 100037, China
}

\begin{abstract}
Background: HOX transcript antisense RNA (HOTAIR), which is expressed from the homebox C gene (HOXC) locus, is capable of reprogramming chromatin organisation and promoting cancer cell metastasis and can simultaneously bind the polycomb repressive complex 2, which enhances H3K27 trimethylation, and the LSD1-CoREST-REST complex, which is critical for H3K4 demethylation. Clinically, the overexpression of HOTAIR is a powerful predictor of the tumour progression and overall survival in patients with diverse cancers. The relationship between HOTAIR and oesophageal squamous cell carcinoma (ESCC), however, remains unclear. We investigated the role of HOTAIR in the pathogenesis of ESCC.
\end{abstract}

\begin{abstract}
Methods: We used quantitative real-time PCR to determine the level of HOTAIR in ESCC cell lines and 100 ESCC samples from patients; 56 adjacent non-neoplastic tissues were used as controls. We measured the effect of HOTAIR knockdown and overexpression in ESCC cell lines using colony formation assays, anchorage-independent growth assays, the CCK-8 assay, transwell migration and invasion assays, and Annexin V-binding assays. We analysed the growth of ESCC xenograft tumours in nude mice. Changes in the gene expression and methylation levels in ESCC cell lines were analysed using gene expression microarrays and the Infinium HumanMethylation450K BeadChip assay, respectively.
\end{abstract}

Results: The levels of HOTAIR were increased in ESCC cell lines and patient samples compared with the controls; the expression levels correlated with the disease stage and survival time. The knockdown of HOTAIR in the KYSE510 and KYSE180 ESCC cell lines using small hairpin RNAs (shRNAs) reduced the ability of the cells to form foci, migrate, and invade the extracellular matrix in culture, altered cell cycle progression, and increased the sensitivity of the cells to apoptosis. The HOTAIR knockdown reduced cancer cell metastasis in vivo, and the tumours formed by HOTAIR-silenced ESCC cells were smaller, both in size and weight, than the tumours and metastases formed by the shRNA vector control cells in a mouse xenograft model. The results of the gene microarray study showed that HOTAIR reprogrammed the gene expression profile of ESCC cells, and the gene ontology analysis revealed an enrichment in genes that are important for tumorigenesis, such as genes involved in cell migration and the regulation of the cell cycle. Comparing the gene expression profiles and DNA methylation analysis between the KYSE180 and KYSE180_HOTAIR cells revealed that only a small proportion of the methylation changes were correlated with gene expression changes.

Conclusion: HOX transcript antisense RNA is upregulated in ESCC cell lines and patient samples, and promotes ESCC cell proliferation and tumour metastasis in mice. The knockdown of HOTAIR resulted in significant changes in gene expression, and data analysis suggested that HOTAIR-mediated gene regulation has a critical role in ESCC progression and is a novel epigenetic molecular target for treating ESCC patients. 
Human oesophageal cancer (EC) occurs worldwide with a variable geographic distribution. The disease ranks ninth in order of occurrence and sixth as the leading cause of cancer mortality, affecting men more than women (Jemal et al, 2011). There are two main forms, each with distinct aetiological and pathological characteristics: oesophageal squamous cell carcinoma (ESCC) and oesophageal adenocarcinoma (EAC; Enzinger and Mayer, 2003). Oesophageal squamous cell carcinoma (SCC) is the most frequent subtype of EC, although the incidence of EACs in the western world is increasing faster than other malignancies. Oesophageal SCC is one of the most aggressive and lethal malignancies in eastern Asia (Enzinger and Mayer, 2003). The prognosis and the overall 5-year survival rate for patients with ESCCs remains extremely grim and very low, mainly because of the advanced stage at initial diagnosis and the deficiency of efficacious therapies (Enzinger and Mayer, 2003; Pohl and Welch, 2005). Recently, the combination of chemotherapy and radiotherapy, alone or as an adjunct to surgery, has improved the prognosis of ESCC patients (Medical Research Council Oesophageal Cancer Working G, 2002). Research over the last two decades has identified a number of oncogenic and tumour suppressor proteins that are associated with the induction of ESCC (Lin et al, 2009). However, molecular indicators of the origin of cellular deregulation in ESCC have not been identified. Elucidation of the molecular pathways involved in ESCC carcinogenesis could lead to improvements in disease diagnosis and therapy. Therefore, to improve outcomes in this prevalent form of cancer, the identification of new molecular markers for early detection and the development of improved prognostic analyses and therapies are urgently required. A better understanding of the recurrent genetic alterations and underlying molecular mechanisms involved in ESCC development and progression will facilitate the identification of novel targets, allowing for more sensitive methods of detection, facilitating earlier diagnosis, and prolonging patient survival.

Mounting evidence indicates that eukaryotic transcriptomes and genomes are not the simple, well-ordered substrates of gene transcription that they were once thought to be. It is now known that genomes are pervasively transcribed and produce a broad spectrum of RNA molecules ranging from long protein-coding mRNAs to short non-coding transcripts whose coding sequences frequently overlap or are interlaced on either strand (Mattick, 2004; Ponting et al, 2009). Until recently, non-coding regions in the genome referred to those that are transcribed into RNA but not translated into protein. These non-coding regions are interspersed throughout the genome. However, the functions of non-coding RNAs (ncRNAs) are only partially understood. It is known that ncRNAs are key factors in gene regulation and influence normal and cancer cell phenotypes (Borchert et al, 2006; Prasanth and Spector, 2007; Carthew and Sontheimer, 2009; Guttman et al, 2011). One subcategory of these transcripts, referred to as microRNAs, has been extensively investigated, and it is estimated that over 1000 microRNAs regulate up to $30 \%$ of all protein-coding genes (Berezikov and Plasterk, 2005; Borchert et al, 2006; Pillai et al, 2007; Carthew and Sontheimer, 2009). Several microRNAs are overexpressed in different tumour types and their functional oncogenic activity is often associated with both the inhibition of genes with tumour suppressor-like activity and the induction of oncogenes (Lu et al, 2005; Nelson and Weiss, 2008; Farazi et al, 2011). Another subcategory of these transcripts, referred to as long ncRNAs (lncRNAs), are typically defined as transcripts that are longer than 200 nucleotides but lack an appreciable open reading frame, which is usually $<100$ amino acids (Mercer et al, 2009). Long ncRNAs are broadly transcribed in the genome, but our understanding of their function is limited. Until recently, lncRNA transcription was believed to represent random transcriptional noise (Ponjavic et al, 2007). However, the expression levels of lncRNA transcripts have been observed to vary spatially, temporally, and in response to various stimuli (Mercer et al, 2008, 2009). There is evidence that many lncRNAs act as scaffolds that regulate the molecular (that is, protein, RNA, and DNA) interactions required for various signalling networks, which is accomplished, in part, via interactions with chromatin-modifying complexes and the epigenetic regulation of the expression of multiple genes (Mercer et al, 2009; Ponting et al, 2009; Li et al, 2013b).

Long ncRNAs are emerging as a novel class of ncRNAs. Several lncRNAs have been identified as being linked to human disease and exerting specific functions (Wapinski and Chang, 2011; Li et al, 2013b). One example of such an oncogenic lncRNA is the HOX transcript antisense RNA (HOTAIR), which is expressed from the HOXC locus but repressed transcription in the more distal HOXD locus in foreskin fibroblasts (Rinn et al, 2007). HOX transcript antisense RNA has been implicated in the development of primary breast tumours and breast cancer metastases, wherein elevation of HOTAIR expression promoted invasion and metastasis (Gupta et al, 2010). The activity of HOTAIR is due, in part, to the interaction of HOTAIR with the polycomb repressive complex 2 (PRC2), which comprises the histone H3K27 methylase EZH2, SUZ12, and EED and enhances H3K27 trimethylation to decrease the expression of multiple genes, especially metastasis-suppressing genes (Rinn et al, 2007; Gupta et al, 2010; Tsai et al, 2010). Recently, HOTAIR was found to be significantly overexpressed in a variety of tumours and was shown to induce the proliferation and metastasis of these tumours (Wapinski and Chang, 2011). Clinically, the overexpression of HOTAIR is a powerful predictor of overall survival and progression for several cancers, including gastrointestinal stromal tumours (Niinuma et al, 2012), colon cancer (Kogo et al, 2011), pancreatic cancer (Kim et al, 2013), hepatocellular carcinoma (Geng et al, 2011; Yang et al, 2011; Ishibashi et al, 2013), nasopharyngeal carcinoma (Nie et al, 2013), and laryngeal SCC (Li et al, 2013a).

The relationship between HOTAIR and ESCC, however, remains limited. Herein, we investigated the expression of HOTAIR in ESCC and explored the clinical significance of HOTAIR expression. We found that HOTAIR was markedly overexpressed in a large cohort of human ESCC samples and was highly expressed in advanced tumours. The inhibition of HOTAIR expression in ESCC cells resulted in diminished cell growth, migration, and invasion, as well as an increase in apoptosis both in vitro and in vivo. To the best of our knowledge, these data establish for the first time that HOTAIR functions as an oncogene in ESCC progression and is a novel epigenetic molecular target for treating ESCC patients.

\section{MATERIALS AND METHODS}

Cells. The EC cell lines TE1, TE3, TE7, TE8, KYSE30, KYSE180, KYSE150, KYSE140, KYSE510, KYSE450, and BIC1 were grown in RPMI-1640 (Invitrogen, Carlsbad, CA, USA) supplemented with $10 \%$ fetal bovine serum (Gibco, Life Technologies, Grand Island,

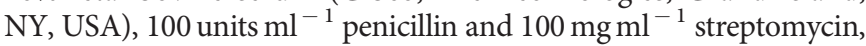
according to the instructions provided. The cultures were maintained at $37^{\circ} \mathrm{C}$ in a humidified atmosphere with $5 \% \mathrm{CO}_{2}$.

Patient information and tissue specimens. This study was conducted using a total of 100 ESCC and 56 adjacent nonneoplastic tissue samples, which were histopathologically and clinically diagnosed at the Chinese PLA General Hospital (Beijing, China). All of the specimens were immediately frozen in liquid nitrogen and stored at $-80^{\circ} \mathrm{C}$ until RNA extraction. Patients had received no previous local or systemic treatment before their operation. Prior patient consent and approval from the institutional research ethics committee was obtained for the use of these clinical materials for research purposes. The clinical information regarding the samples is summarised in Table 1. 
Additional details of biochemical and cellular reagents, primers, and protocols are described in Supplementary Materials and Methods.

Statistical analysis. Statistical tests for data analysis included the Fisher exact test, log-rank test, $\chi^{2}$-test, and Student's two-tailed $t$-test. Multivariate statistical analysis was performed using a Cox regression model. The statistical analyses were performed using the SPSS 11.0 statistical software package (SPSS Inc., Chicago, IL, USA). The data represent the mean \pm s.d. $P$-values $\leqslant 0.05$ were considered to be statistically significant.

\section{RESULTS}

HOX transcript antisense RNA overexpression correlates with cancer progression and poor prognosis in human ESCC. To investigate the oncogenic role of HOTAIR in ESCC progression, we examined the expression of HOTAIR in human ESCC tissues and ESCC cell lines by quantitative real-time PCR (qRT-PCR). As shown in Figure $1 \mathrm{~A}$ and $\mathrm{B}$, comparative analyses showed that HOTAIR was differentially elevated in 100 human ESCC samples compared with the 56 adjacent non-neoplastic tissues. HOX

Table 1. Clinicopathological characteristics of studied patients and expression of HOTAIR in ESCC

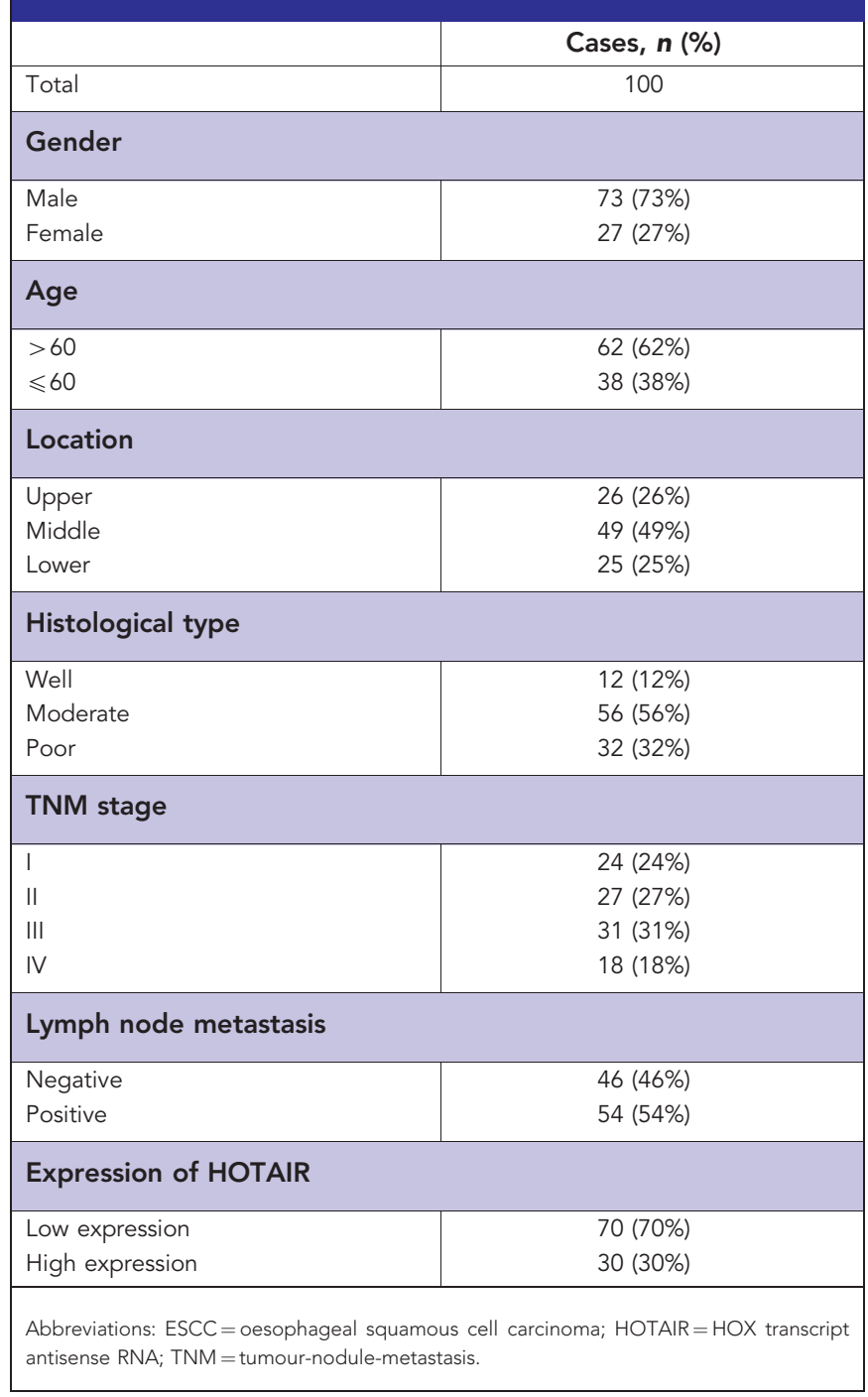

transcript antisense RNA was upregulated in all of the 11 analysed ESCC cell lines compared with the 9 primary normal oesophageal epithelial cell lines (NEECs). These results suggest that HOTAIR is overexpressed in ESCC.

The multivariate analyses revealed that HOTAIR expression strongly correlated with the clinical stage, tumour-nodule-metastasis classification, lymph node metastasis, and histological differentiation in patients with ESCC, suggesting that HOTAIR overexpression is associated with the clinical progression and development of human ESCC (Figure 1C and D). Moreover, we found that the survival time of the ESCC patients with high levels of HOTAIR expression was significantly different compared with those with low levels of HOTAIR. On the basis of the expression levels of HOTAIR obtained by quantitative PCR, we divided the 100 ESCC patients into a high-HOTAIR expression group $(n=30$, $>125$-fold compared with NEECs) and a low-expression group $(n=70$; Figure $1 \mathrm{E})$. The Kaplan-Meier survival analysis and logrank tests using patient postoperative survival were conducted to further evaluate the correlation between HOTAIR expression and the prognosis of patients with ESCC. From the Kaplan-Meier survival curve, we observed that patients with high levels of HOTAIR expression $(n=30)$ had significantly shorter survival times than those with low levels of HOTAIR expression $(n=70$, hazards ratio $=1.913,95 \% \mathrm{CI}=1.06-3.997, P=0.0334$, log-rank test; Figure $1 \mathrm{~F})$. These results suggest that the HOTAIR expression level can be employed as a powerful independent prognostic factor. Thus, we concluded that HOTAIR has potential clinical value as a predictive biomarker for disease outcome in ESCC.

HOX transcript antisense RNA promotes the aggressiveness of ESCC cells ex vivo. To investigate whether HOTAIR has a role in the pathogenesis of ESCC, KYSE510, and KYSE180, ESCC cell lines were established that displayed a stable knockdown of HOTAIR expression (Figure 2A). Strikingly, the silencing of HOTAIR expression significantly reduced both the anchorageindependent and -dependent growth ability of the cells (Figure 2B and $\mathrm{C}$ ) and led to lower growth rates (Figure 2D) compared with cells transduced with a control small hairpin RNA (shRNA). We also investigated whether HOTAIR knockdown has an inhibitory effect on ESCC cell invasion. The stable knockdown of HOTAIR in the KYSE510 and KYSE180 cells led to a significant decrease in the ability of the cells to invade through an extracellular matrix (Figure 2E).

To explore the potential mechanism that underlies the growth inhibitory activity of HOTAIR, we performed flow cytometry to compare the DNA content between HOTAIR-repressed and control KYSE180 cells. The results showed that the cell population in the G1 phase was increased but the S-phase population was decreased after the depletion of HOTAIR compared with the results seen in the control cells (Figure $2 \mathrm{~F}$, top), suggesting that HOTAIR may affect the G1/S transition. To better understand the function of HOTAIR in the G1/S transition, cell cycle distribution analyses were conducted in the presence of nocodazole, which blocks cells in mitosis (Zieve et al, 1980). After treatment with nocodazole for $8 \mathrm{~h}$, a marked increase in the G1 population and decrease in the G2/M population were observed in HOTAIR knockdown cells compared with the control shRNA cells (Figure 2F, bottom). Thus, HOTAIR might be essential for the progression of an orderly G1/S transition. The potential role of HOTAIR in tumour progression was evaluated by examining the impact of HOTAIR on apoptosis in ESCC cells. Consistent with the results of previous studies on HOTAIR (Kim et al, 2013), when the KYSE510 and KYSE180 cells were transiently transfected with HOTAIR siRNA using Lipofectamine 2000 (Invitrogen), which effectively knocked down the expression of HOTAIR within $48 \mathrm{~h}$, a significant increase in apoptosis was observed in the KYSE510 and 
A

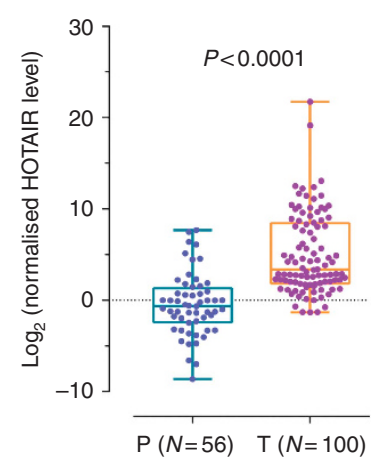

D

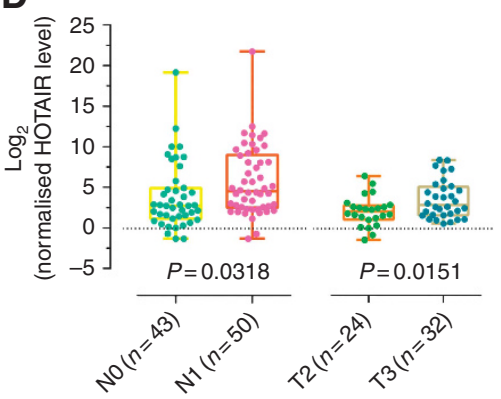

B

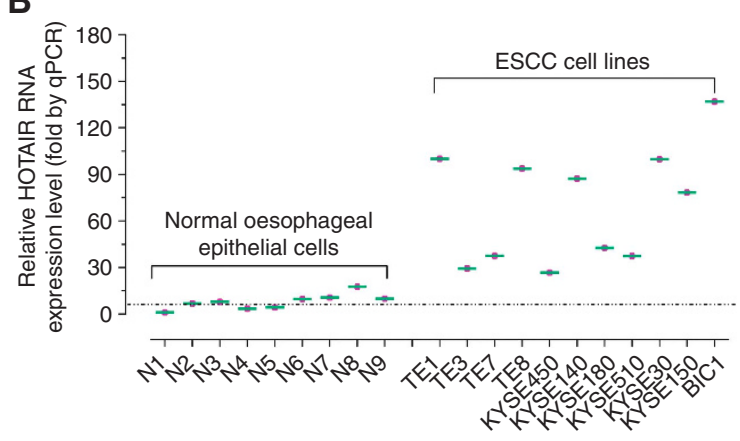

E

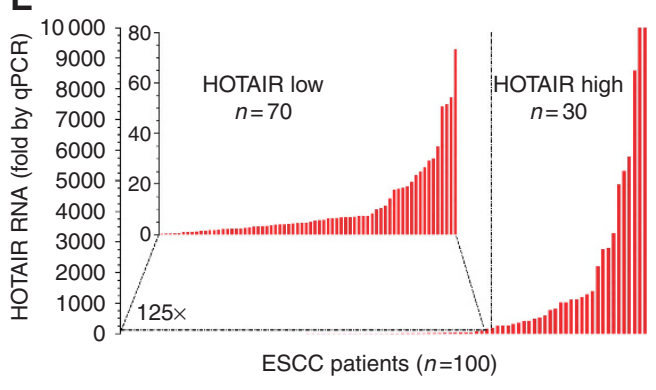

C

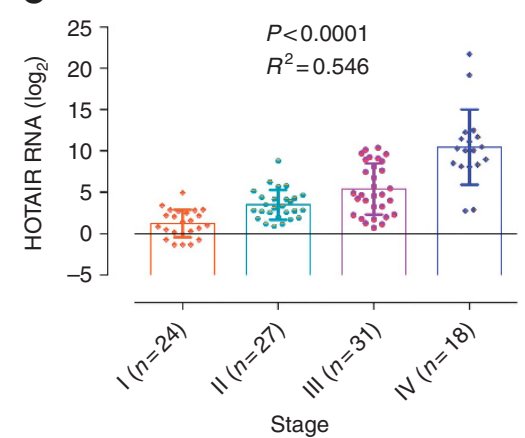

$\mathbf{F}$

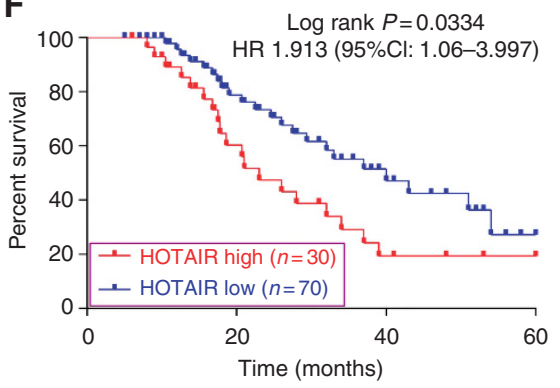

Figure 1. High levels of HOTAIR are correlated with progression and prognostic significance in ESCC. (A) HOX transcript antisense RNA expression in normal oesophageal epithelial tissues and ESCC tissues from patients. The majority of cases exhibited elevated levels of HOTAIR in tumours compared with levels observed in noncarcinoma tissues $(P<0.001)$. Relative gene expression determinations were made with the comparative delta-delta CT method $\left(2^{-\Delta \Delta C t}\right)$. (B) The relative expression levels of HOTAIR were confirmed via real-time PCR in 11 EC cell lines. (C) HOTAIR levels categorised according to tumour-nodule-metastasis stage. (D) Oesophageal SCC patients with lymph node metastasis displayed significantly higher HOTAIR expression levels $(P<0.05)$. (E) The total of 100 ESCC patients included in this study were divided into an elevated HOTAIR expression group $(n=30)$ and a low HOTAIR expression group $(n=70)$ the value of relative HOTAIR expression (125-fold). (F) The Kaplan-Meier overall survival curves by HOTAIR levels. Patients with elevated HOTAIR expression $(n=30)$ showed reduced survival times compared with patients with low levels of HOTAIR expression $(n=70$; log-rank test; $P=0.0334$ ).

KYSE180 cells compared with cells infected with the control shRNA vector (Figure $2 \mathrm{G}$ ).

To further assess the role of HOTAIR in the pathogenesis of ESCC, KYSE510, and KYSE180, ESCC cell lines stably expressing ectopic HOTAIR were established (Figure 3A). Consistent with the previous results, the ectopic expression of HOTAIR led to a significant increase in both anchorage-independent and -dependent growth (Figure $3 \mathrm{~B}$ and $\mathrm{C}$ ) and promoted the overall growth rate of the cells (Figure 3D). We also investigated whether the overexpression of HOTAIR affected ESCC cell invasion and migration. Stable HOTAIR overexpression in both KYSE510 and KYSE180 cells resulted in a significant increase in the ability of the cells to migrate (Figure 3E) and invade through an extracellular matrix (Figure $3 F$ ). In addition, we performed flow cytometry to compare the DNA content between the HOTAIR-overexpressed and control cells. The results showed that the proportion of S-phase cells was significantly greater in HOTAIR-overexpression clones (38.36\% in KYSE510 cells; $41.03 \%$ in KYSE180 cells) compared with empty vector cells $(31.00 \%$ in KYSE510 cells; $28.99 \%$ in KYSE180 cells; Figure 3G). Collectively, these results suggest that HOTAIR has an important role in the tumorigenicity of ESCC cells ex vivo.

HOX transcript antisense RNA contributes to the progression of ESCC in vivo. The ability of HOTAIR to promote ESCC progression was examined using an in vivo tumour model. We generated KYSE180 ESCC cells with stable HOTAIR knockdown using a shRNA lentiviral knockdown system. More than $90 \%$ of KYSE180 cells expressed GFP at $72 \mathrm{~h}$ after lentiviral transduction, indicating that there was an efficient and stable transduction of the lentiviral vector (Supplementary Figure 1A). Quantitative real-time
PCR was performed to confirm that there was an efficient depletion of HOTAIR expression. HOX transcript antisense RNA was expressed at a significantly lower level in KYSE180 cells transduced with the HOTAIR shRNA lentivirus than in cells transduced with the GFP lentivirus, indicating that the HOTAIR shRNA effectively decreased HOTAIR expression (Supplementary Figure 1B). To quantify the metastatic potential of the HOTAIRknockdown cells in vivo, we performed tail vein injections and compared the rates of lung colonisation by the normal and HOTAIR-knockdown cells. Consistent with a previous study of HOTAIR in breast cancer (Gupta et al, 2010), the stable knockdown of HOTAIR in ESCC cells significantly suppressed their metastasis to the lung (Figure 4Aa). Haematoxylin-eosin staining of lung sections from mice injected with the HOTAIRsilenced cells confirmed that HOTAIR knockdown significantly inhibited the colonisation of cells in the lung (Figure 4Ab). The tumours formed by the HOTAIR-silenced cells were smaller both in size and weight than the tumours formed by the cells expressing the control shRNA vector (Figure $4 \mathrm{Ba}$ and $\mathrm{Bb}$ ). Immunohistochemical analysis showed that tumours derived from the HOTAIR-silenced cells displayed a decreased microvascular density, a lower Ki67 proliferation index, and a higher percentage of TUNEL-positive apoptotic cells compared with the tumours derived from cells infected with the control shRNA vector (Figure 4C). Taken together, these results indicate that HOTAIR contributes to the progression of ESCC in vivo.

HOX transcript antisense RNA reprogrammes the gene expression in ESCC cells. We validated the HOTAIR-regulated gene expression profile that could contribute to the functional oncogenic activity of HOTAIR in ESCC cells. To identify the molecular 
changes that are regulated by HOTAIR, comparative microarray analysis was performed on RNA isolated from KYSE180 ESCC cells with and without stable HOTAIR repression. To avoid off- target effects, the HOTAIR knockdown was performed using two different siRNAs (siHOTAIR I and siHOTAIR II). The microarray analysis revealed that the knockdown of HOTAIR by RNAi
A

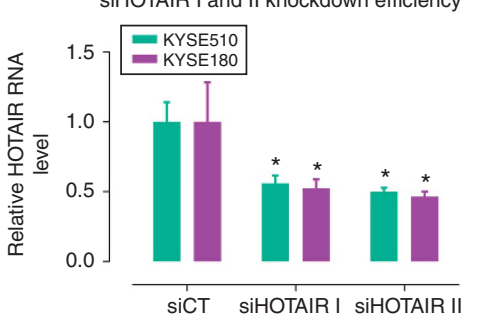

C
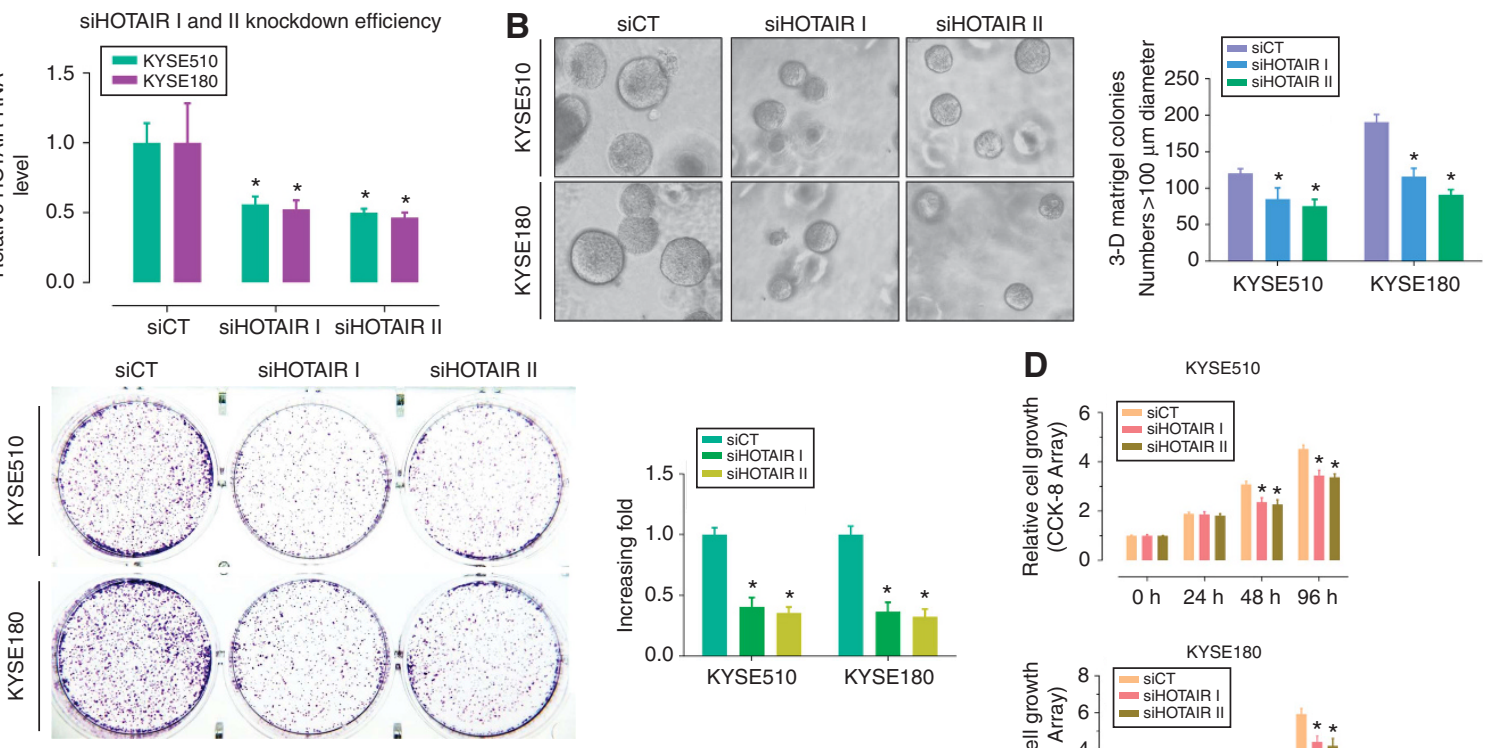

D
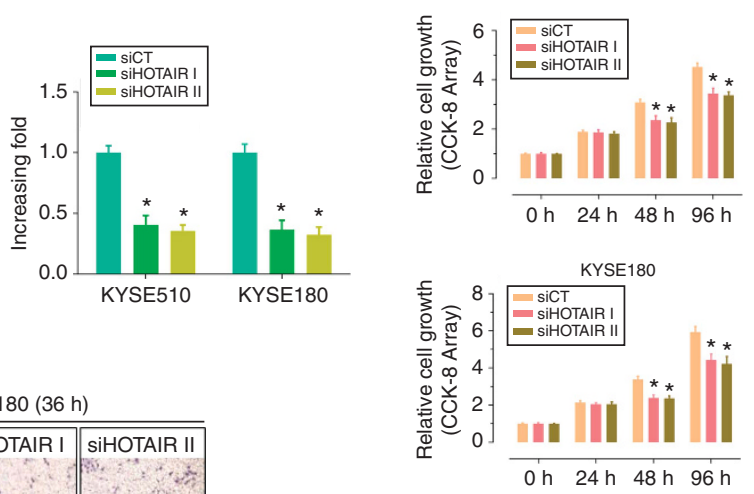

KYSE180 (36 h)
E

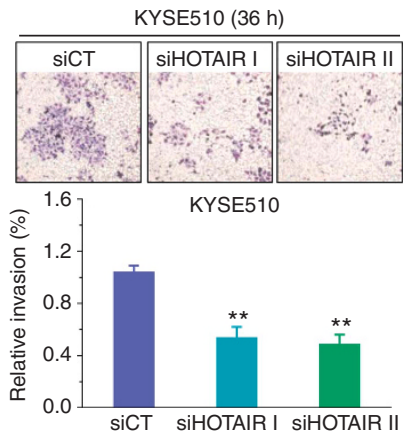

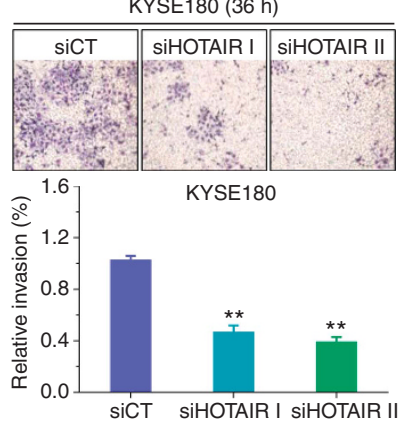

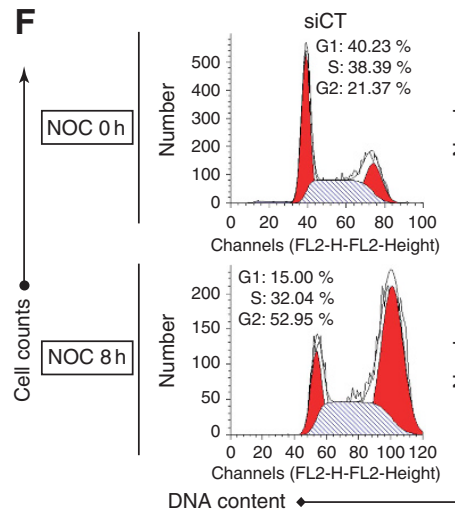

G

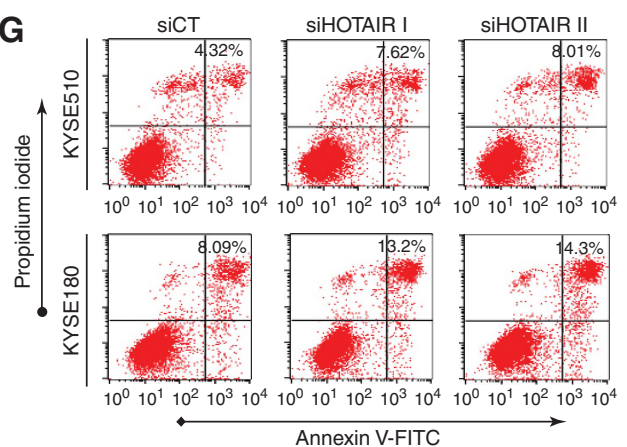

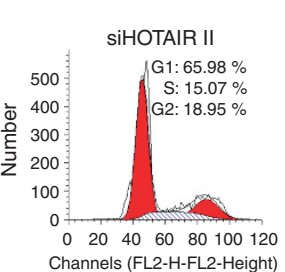
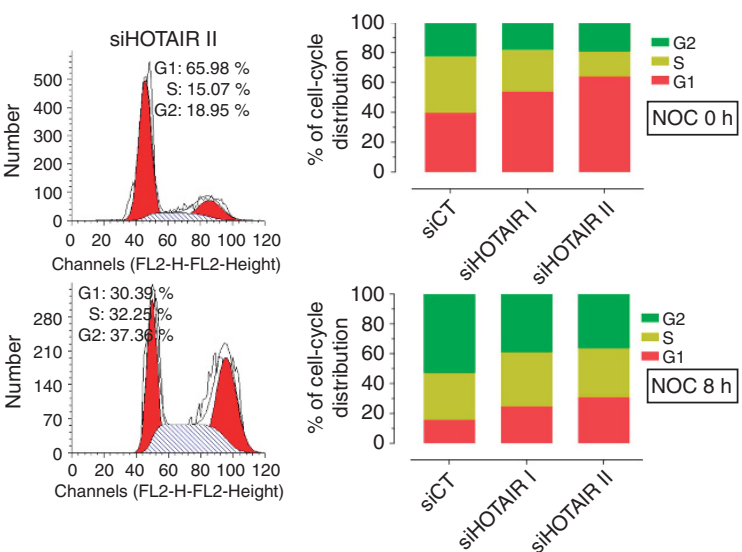

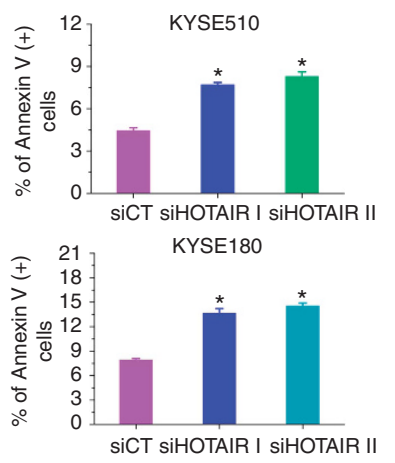


(siHOTAIR) resulted in changes in the expression of 2853 genes (fold change >two-fold); the expression of 2458 genes was upregulated ( $A$ regional, Supplementary Figure 2) and the expression of 395 genes was downregulated ( $B$ regional, Supplementary Figure 2). A visualisation of the differential expression pattern for these 2853 genes is shown in Figure 5A using a hierarchical clustering heat map. A representative list of these genes along with their accession numbers, signal values, and average fold change is shown in Supplementary Table 1 and 2. Gene ontology (GO) analysis was performed using GOStat (Beissbarth and Speed, 2004) to study the biological function of the 2853 genes differentially expressed in the KYSE180 HOTAIR knockdown cells compared with the control (siCT) cells (Supplementary Table 3). A selection of significant GO terms for biological processes and molecular functions is shown in Figure $5 \mathrm{~B}$ and C. Consistent with our previous functional studies, most of the GO terms were related to tumorigenesis, including apoptosis, cell migration, DNA replication and repair, cell cycle regulation, and response to DNA damage stimulus. The same gene set was surveyed using the Kyoto Encyclopedia of Genes and Genomes pathway database, and several significantly enriched pathways were identified that corresponded to the genes with the greatest transcriptional variation (Supplementary Table 4). A selection of critically overrepresented pathways is provided in Figure 5D; pathways relating to apoptosis and cell adhesion are well represented among the deregulated genes.

HOX transcript antisense RNA preferentially selects for DNA hypermethylation in KYSE180 cells. We used the KYSE180 cell lines stably expressing either ectopic HOTAIR or control vectors to investigate the role of HOTAIR in DNA methylation changes that are associated with changes in gene expression. We performed genome-wide DNA methylation profiling of KYSE180 cells stably expressing ectopic HOTAIR and the control cells using the Infinium HumanMethylation450K BeadChip (Illumina, San Diego, CA, USA), which interrogates over 480000 of the 28 million CpG sites in the human methylome across $>20000$ genes. Supplementary Figure 3 summarises the genomic environment of the 485145 CpGs.

Before analysing the $\mathrm{CpG}$ methylation data, we excluded possible sources of technical bias that could have influenced the results. Every $\beta$-value in the Infinium HumanMethylation450K BeadChip platform is accompanied by a detection of $P$-value. We found that a threshold $P$-value above 0.01 indicated unreliable $\beta$-values in $608 \mathrm{CpGs}(0.125 \%)$ of the 485577 sites analysed. Thus, using this filter, $484969 \mathrm{CpGs}$ proved to be reliable and were used subsequently in the study.

As shown in Figure 5E, KYSE180 cells had 285062 CpGs that were methylated over a basal $\beta$-value of 0.6 , whereas KYSE180_HOTAIR cells showed a $1.4 \%$ increase in CpG methylation. These data suggest that HOTAIR has a role in maintaining the DNA methylation status of these CpG sites. We analysed KYSE180 cells in comparison with KYSE180_HOTAIR cells at each CpG position.
We extracted the differentially methylated genes that showed a significantly increased or decreased $\beta$-difference $(|\Delta \beta|)$ at a minimum of one $\mathrm{CpG}$ site; this corresponded to a false discovery rate of $<0.05$ that was estimated using biological replicates within the study $(|\Delta \beta| \sim 0.1$ ), as described previously (Zeller et al, 2012). Using these criteria, we identified multiple methylation differences between KYSE180_HOTAIR and KYSE180 cells, suggesting that HOTAIR could preferentially select for DNA methylation in KYSE180 ESCC cells (Figure 5F). Of the 484969 CpGs studied, significant DNA methylation differences were observed between the KYSE180_HOTAIR and KYSE180 cells at $1.54 \%$ of the sites (7475 CpGs; Figure 5G). Most importantly, 80\% (5989 CpGs) of the observed differential DNA methylation changes corresponded to a CpG hypermethylation event in the KYSE180_HOTAIR cells, whereas hypomethylation changes only accounted for 20\% (1486) of the total observed changes in the KYSE180_HOTAIR cells, suggesting that hypermethylation occurs more frequently than hypomethylation after stable expression of ectopic HOTAIR (Figure 5G). The difference between the gain and loss of DNA methylation was not only quantitative but also qualitative. These differential CpG methylation events were widely distributed throughout the genome of KYSE180_HOTAIR cells and

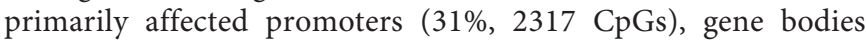
(27\%, $2018 \mathrm{CpGs)}$, and intergenic regions (25\%, $1869 \mathrm{CpGs}$; Figure $5 \mathrm{H})$. There was no association between the differential DNA methylation events and the chromosome location; both hyper and hypomethylation changes occurred at all $\mathrm{CpG}$ sites (Supplementary Figure 4), and all chromosomes displayed both types of DNA methylation events (Figure 5I and Supplementary Figure 3B).

Methylation changes in ectopic HOTAIR-expressing cells is associated with gene expression changes in only a subset of genes. As aberrant changes in DNA methylation at $\mathrm{CpG}$ islands have been directly linked to changes in gene expression and are strongly associated with transcriptional repression (Jones and Baylin, 2002; Esteller, 2008), we were interested in whether the changes in the DNA methylome were mirrored on a functional level in the ESCC transcriptome. We assumed that the expression changes associated with hypermethylation in KYSE180_HOTAIR cells compared with the control cells would probably represent silencing events associated with the malignant phenotype. To gain an overall perspective on the relationship between differential methylation and expression, we examined the mRNA expression profiles in the KYSE180 and KYSE180_HOTAIR cell clones using the Affymetrix GeneChip PrimeView Human Gene Expression Array (Affymetrix, Santa Clara, CA, USA) and identified differentially expressed genes. We screened for genes where hypermethylation was associated with reduced expression in KYSE180_HOTAIR cells. Of 4129 genes hypermethylated in the KYSE180_HOTAIR cells, 4016 (97\%) genes were present on the Affymetrix GeneChip PrimeView Human Gene Expression Array.

Figure 2. Silencing HOTAIR inhibits the malignant properties of ESCC cells. (A) Silencing HOTAIR in two specific short hairpin RNA-transduced stable ESCC cell lines. Relative gene expression determinations were made with the comparative delta-delta CT method ( $\left.2^{-\Delta \Delta C t}\right)$. (B) The representative pictures (left panel) and quantification (right panel) of colony numbers of indicated cells as determined by an anchorageindependent growth assay. Colonies larger than $100 \mu \mathrm{m}$ in diameter were scored. (C) The representative pictures (left panel) and quantification (right panel) of Giemsa-stained cells. (D) The ESCC KYSE510 or KYSE180 cells were transfected with control siRNA or two individual siRNAs against HOTAIR as indicated. Cell viability was detected at the indicated time points after transfection using CCK-8 assays. (E) The representative pictures (top panel) and quantification (bottom panel) of invaded cells were analysed using the transwell matrix penetration assay. (F) KYSE180 cells stably transfected with the indicated siRNAs were treated with Nocodazole (Noc) for $0 \mathrm{~h}$ (top panel), $8 \mathrm{~h}$ (bottom panel). Cell cycle distribution was measured by propidium iodide (PI) staining followed by flow cytometry. The percentage of cells in G1, S, or G2 phase transfected with control siRNA is defined as control. Data are represented as mean \pm s.d. from three independent experiments. (G) Annexin V-fluorescein isothiocyanate (FITC)/PI staining of cells transfected with indicated siRNA for $48 \mathrm{~h}$. Each bar represents the mean $\pm \mathrm{s}$.d. of three independent experiments. ${ }^{\star} P<0.05 ;{ }^{\star *} P<0.01$. 
To analyse the results, we used a scatter plot to compare the relative expression of each gene with the change in its methylation status (Figure 5J). The combined analysis revealed that in KYSE180 cells stably overexpressing HOTAIR, only a small proportion of the methylation changes correlated with gene expression changes; 97 genes were hypermethylated and downregulated and 105 genes
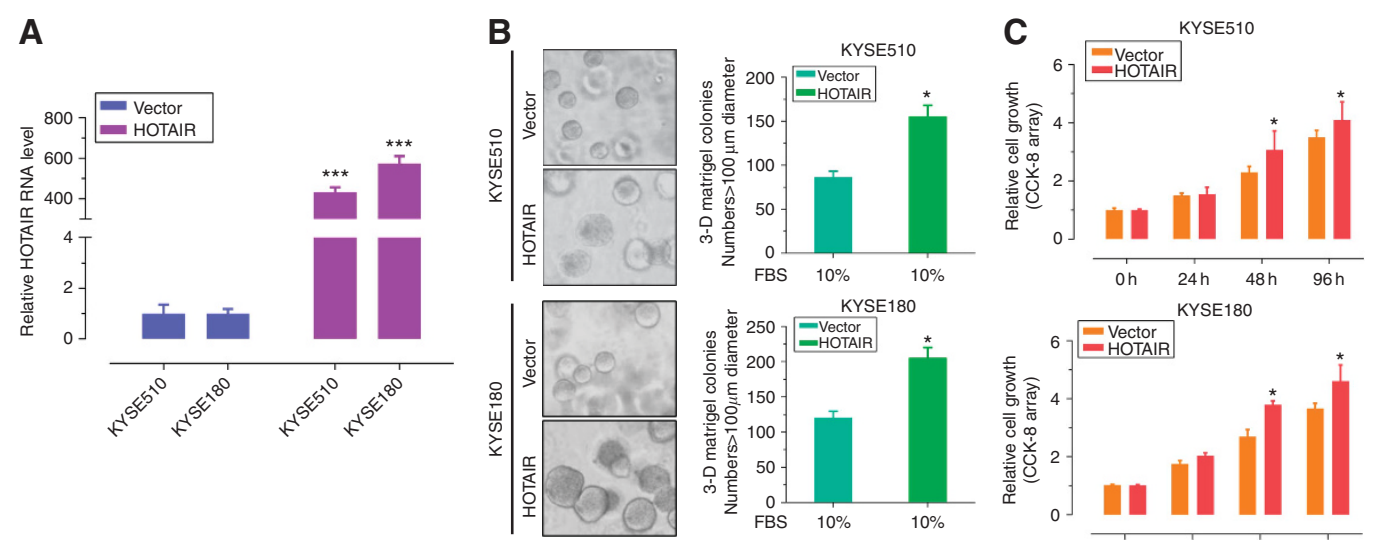

D
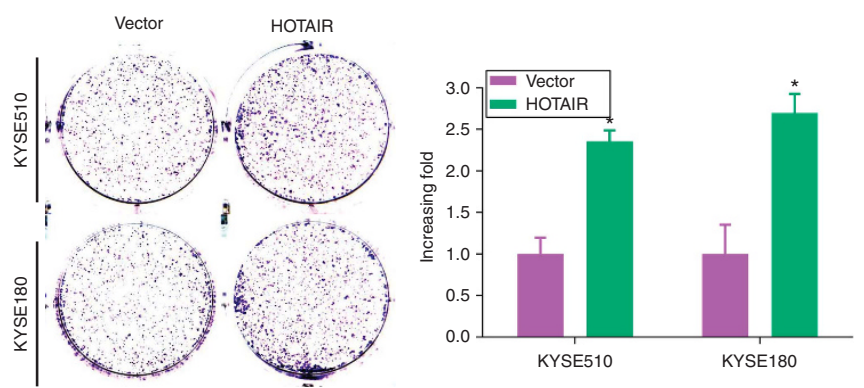

E
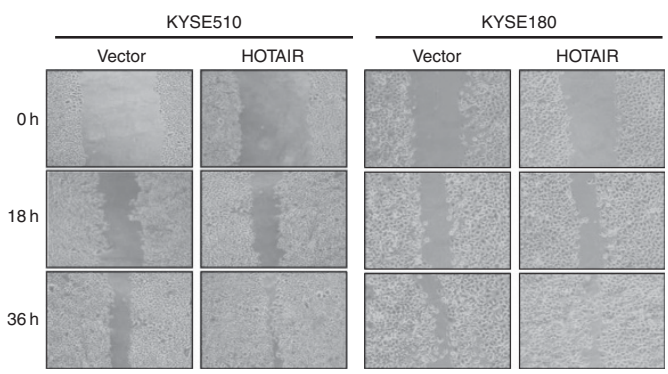

$\mathbf{F}$
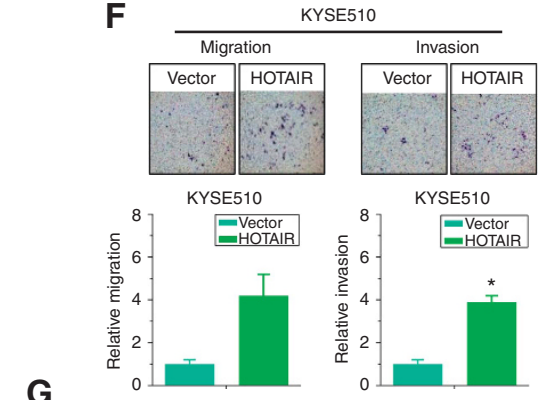

G
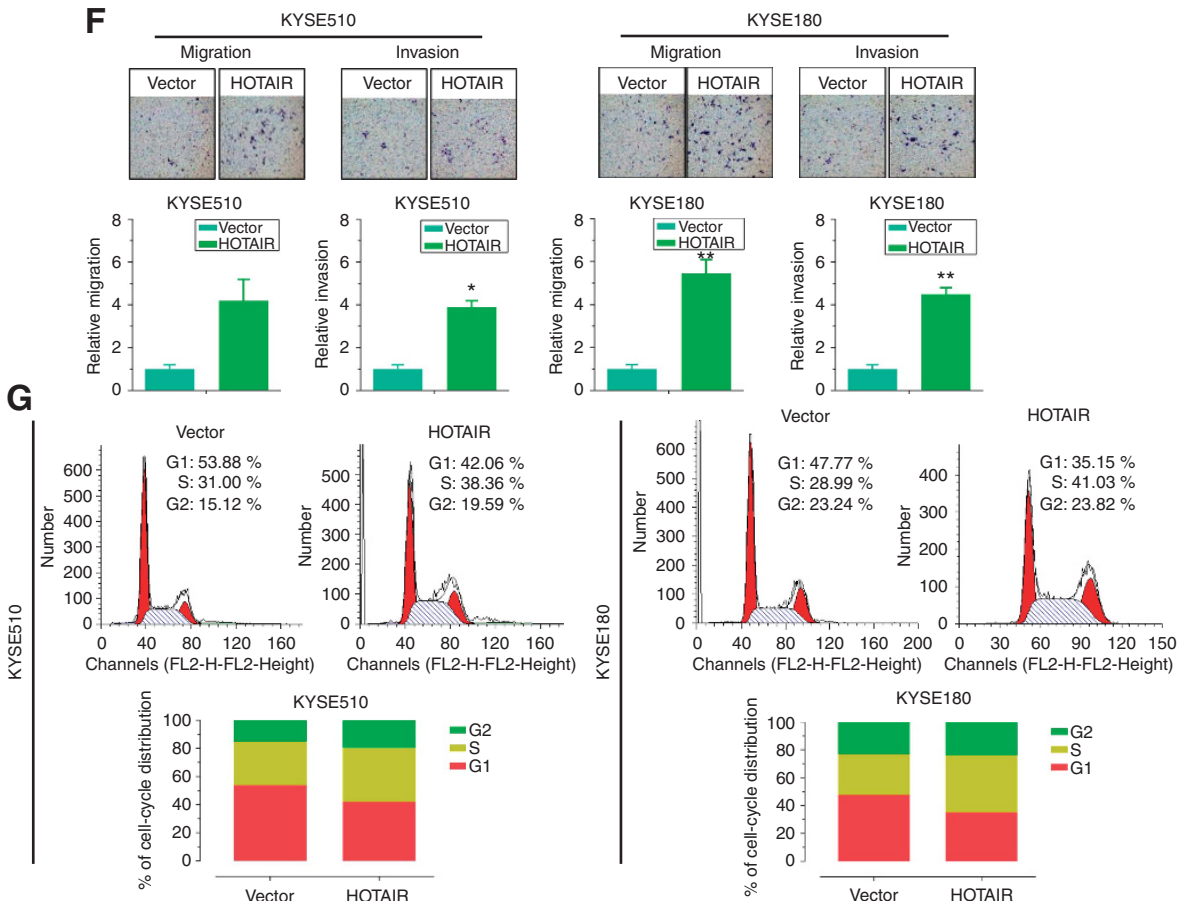
A

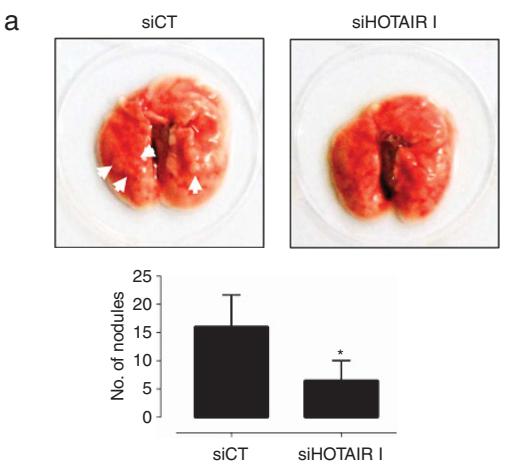

b

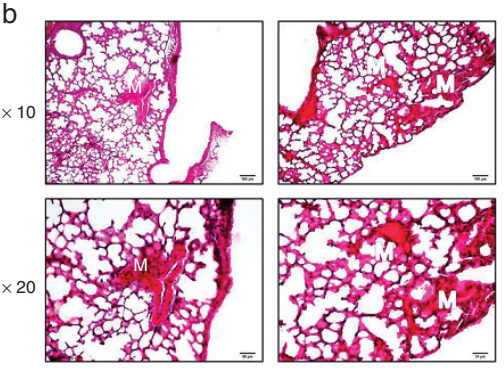

C
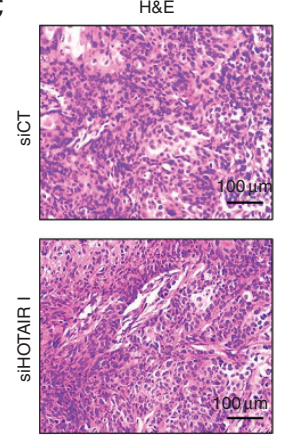

Ki67
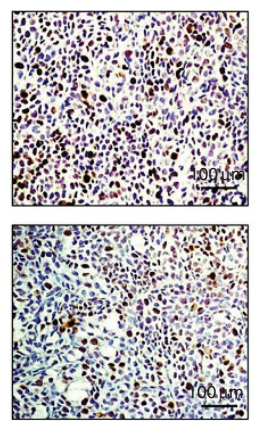

B

a
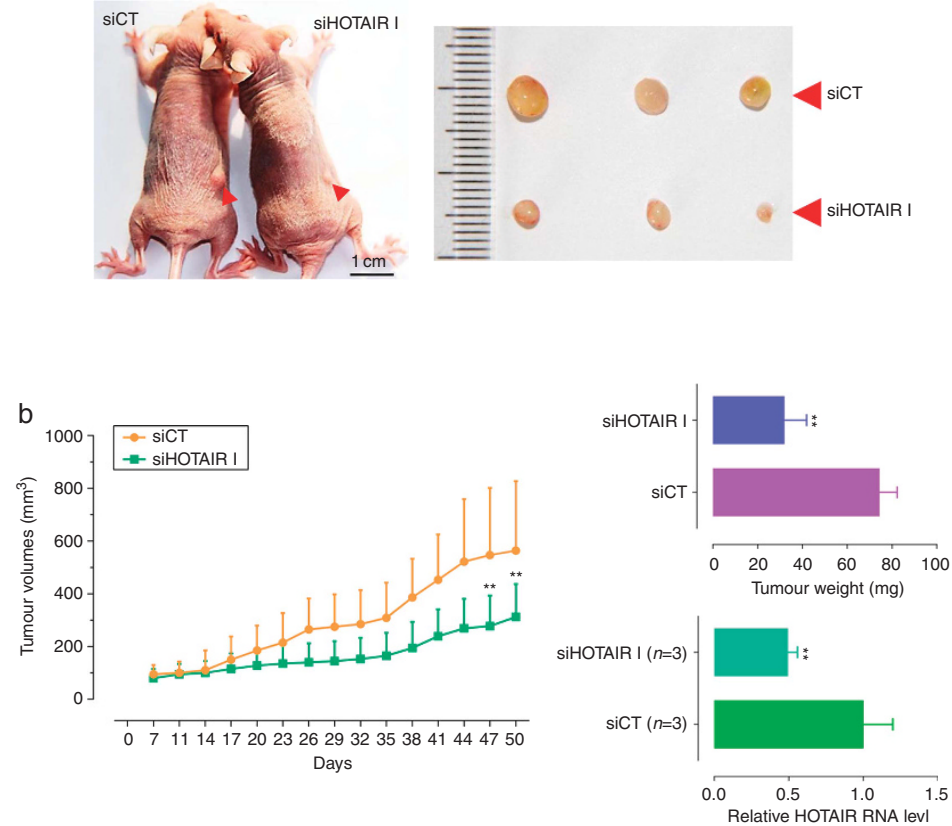

TUNEL

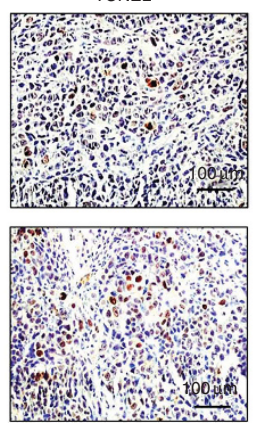

CD31/PECAM1

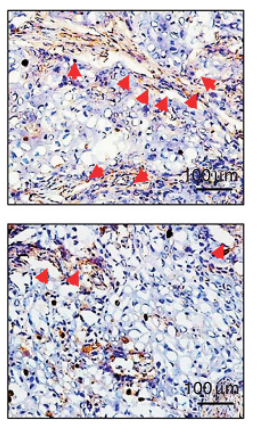

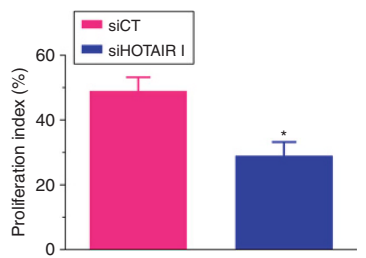
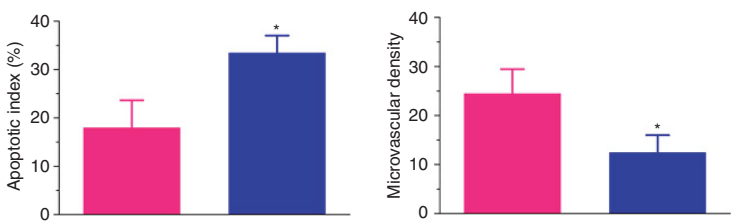

Figure 4. HOX transcript antisense RNA contributes to ESCC progression in vivo. (A) Lung metastasis assay using an intravenous cancer mouse model and histological analysis from nude mice injected via the lateral tail vein with KYSE180 cells with control siRNA or siRNAs against HOTAIR (a). Representative Haematoxylin-eosin (H\&E) images in lung metastases are shown (b). (B) Representative images of tumour-bearing mice (left panel; a) and images of the tumours from all mice in each group (right panel; a). Tumour volumes were measured on the indicated days (left panel; b), and tumour weights were determined (right panel; b). (C) H\&E and immunohistochemical staining demonstrated that suppression of HOTAIR inhibited the aggressive phenotype of ESCC cells in vivo, as indicated by the expression of Ki67-, TUNEL-, and CD31/PECAM1-positive cells. ${ }^{\star} P<0.05 ;{ }^{* \star} P<0.01$.

Figure 3. HOX transcript antisense RNA promotes the aggressiveness of ESCC cells in vitro. (A) Overexpression of HOTAIR in KYSE510 and KYSE180 cell lines analysed by qRT-PCR. Relative gene expression determinations were made with the comparative delta-delta CT method $\left(2^{-\Delta \Delta C t}\right)$. (B) The representative pictures (left panel) and quantification (right panel) of colony numbers of indicated cells as determined by an anchorage-independent growth assay. Colonies larger than $100 \mu \mathrm{m}$ in diameter were scored. (C) Cell viability was detected at the indicated time points after transfection using CCK-8 assays. (D) The representative pictures (left panel) and quantification (right panel) of Giemsa-stained cells. (E) Scratch wound assay results. Overexpression of HOTAIR in KYSE510 and KYSE180 cells produced a higher scratch closure rate than observed in controls infected with empty vector. (F) The representative pictures (top panel) and quantification (bottom panel) of migrated and invaded cells were analysed using transwell matrix penetration assay. (G) Comparison of DNA content between empty vector and HOTAIR overexpressed in both KYSE510 and KYSE180 cell lines by flow cytometry. Summary of cell proportions in different phases of cell cycle is shown. The results are expressed as means \pm s.d. of three independent experiments. ${ }^{\star} P<0.05 ;{ }^{\star \star} P<0.01$; ${ }^{\star \star \star} P<0.001$. 
displayed the opposite pattern of methylation and expression (Supplementary Table 5, Figure 5J and K).

To confirm the relationship between HOTAIR-dependent DNA methylation and expression changes, we selected 11 genes $(A B L 2$, LAMB3, LAMC2, SNAI1, JAM2, PCDH10, PCDHB5, MX1, OAS-1, IFTM1, and GDF15) that have been shown to be regulated by HOTAIR in various cancers. These genes are also included in our
Infinium HumanMethylation450K array. Applying a cutoff of $|\Delta \beta| \geqslant 0.1$, we observed that 4 out of the 11 genes (LAMB3, JAM2, $M X 1$, and $O A S-1)$ acquired methylation and 2 out of the 11 genes (ABL2 and IFTM1) exhibited decreased methylation in KYSE180 cells stably overexpressing HOTAIR (Supplementary Figures 5A and $\mathrm{B}$ ). To determine whether the changes in DNA methylation were associated with changes in gene expression, we measured
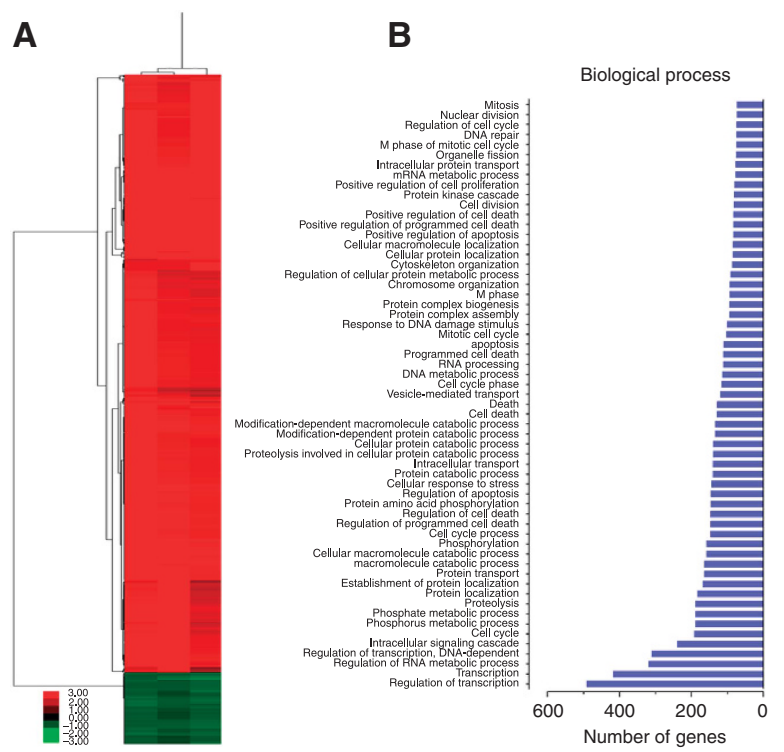

C

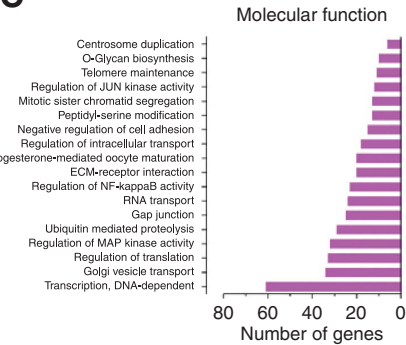

D

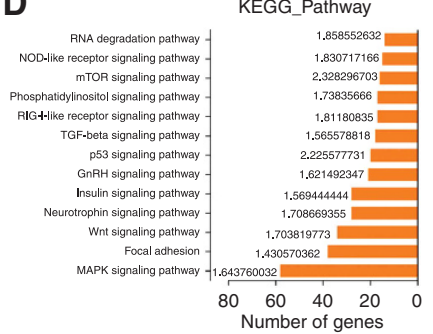

E
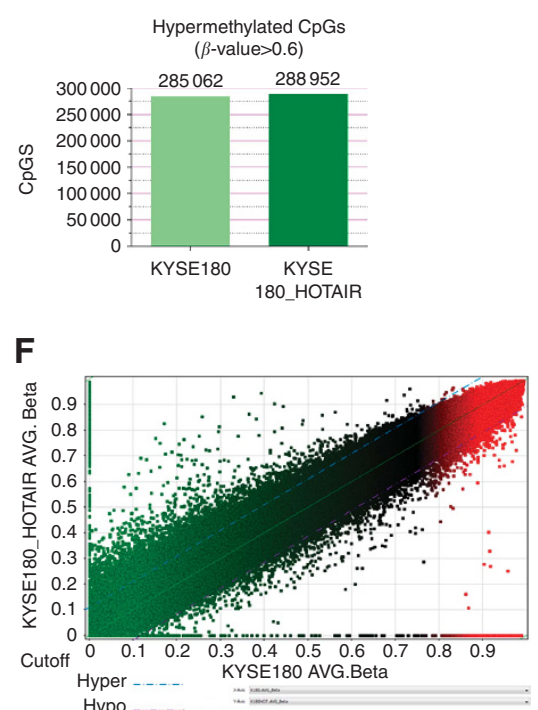

G

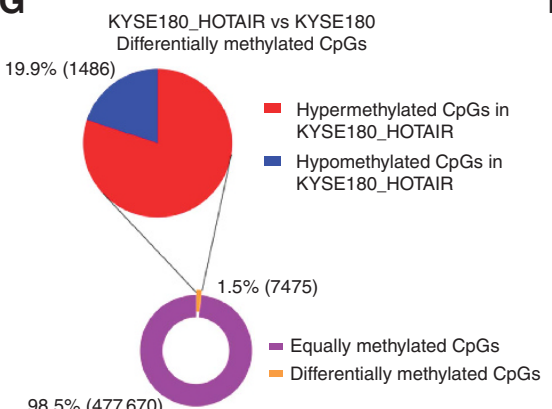

KYSE180_HOTAIR vS KYSE180

$\mathbf{J}$

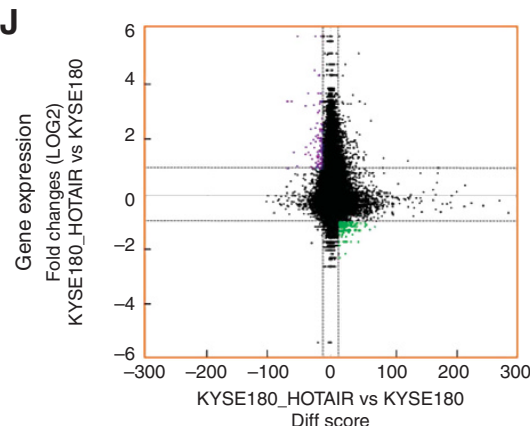

Diff score
H

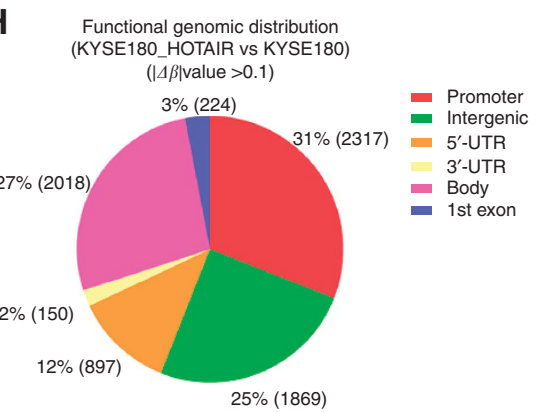

K
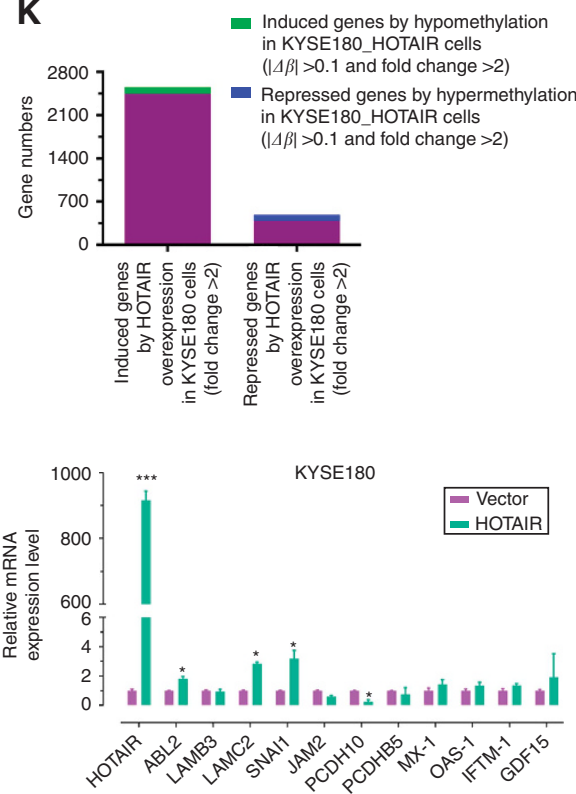

I

Hypermethylated CpGs in KYSE180_HOTAIR Chromosome location $\Delta \beta$ value $>0.1$

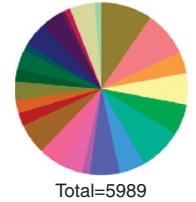

Hypomethylated CpGs in KYSE180_HOTAIR Chromosome location $\Delta \beta$ value $<-0.1$

$\square$ Chr $1-\operatorname{Chr} 13$ $\square$ Chr 2 Chr 14 Chr 3 Chr 15 $\square \operatorname{Chr} 4=\operatorname{Chr} 16$ Chr 5 Chr 17 Chr 18 - Chr 7 豆 $\mathrm{Chr} 19$ Chr 8 Chr 20 $\checkmark$ Chr 9 Chr 21 $\square$ Chr $10 \square$ Chr 22 $\square \mathrm{Chr} 11 \square \mathrm{ChrX}$ $\square$ Chr $12 \square \operatorname{ChrY}$

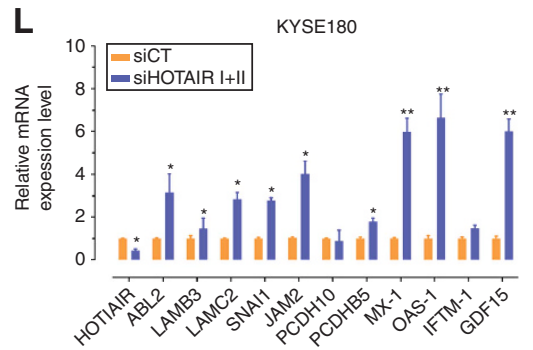


the transcript levels using qRT-PCR. Consistent with the results obtained in our gene expression and methylation microarrays, there was one set of genes (LAMB3, JAM2, MX1, and OAS-1) that showed both increased methylation in the region downstream of the gene and decreased expression in the KYSE180_HOTAIR cells; these genes become re-expressed following HOTAIR knockdown, suggesting that the hypermethylation of these four genes is associated with the downregulation of their expression in KYSE180_HOTAIR cells (Figure 5L and Supplementary Figure 5B). However, the other seven genes are not regulated by HOTAIR-mediated DNA methylation changes in KYSE180 cells (Figure 5L and Supplementary Figure 5B). These data indicate that screening for genes with methylation changes using our method of combining expression microarray data and qRT-PCR data in established cell lines can identify genes whose expression correlates with methylation-regulated transcription. This method identified a small number of genes in KYSE180 ESCC cells for which this is true. The epigenetic regulation of additional differentially expressed genes in ESCC cells might influence HOTAIR oncogenic activity through unknown mechanisms, such as chromatin remodelling. Further studies, including genome-wide histone modification analysis, may reveal as-yet unidentified roles for HOTAIR in the malignant progression of ESCC.

\section{DISCUSSION}

Oesophageal SCC accounts for $>90 \%$ of EC cases and has been ranked the sixth leading cause of cancer-related deaths worldwide (Enzinger and Mayer, 2003; Jemal et al, 2011). Despite the development of multifarious therapies, ESCC patient prognosis remains poor, even for those who undergo a complete resection of the tumour (Allum et al, 2009; Rizk et al, 2010). The limited improvement in the treatment outcome provided by conventional therapies has prompted us to seek innovative, molecularly targeted strategies for treating ESCCs. The identification of new prognostic molecular markers for ESCC may improve the current riskadapted therapeutic strategies and help stratify patients in clinical trials of molecularly targeted drugs (Hildebrandt et al, 2009).

The challenge for clinicians and oncologists in terms of patientpersonalised medicine and plan for treatment is that early-stage tumours with similar histopathological features may subsequently display dramatically different outcome. In this paper, we described the first evidence that oncogene HOTAIR expression in early-stage ESCC negatively correlated with overall postoperative survival of patients. In light of our in vitro data that knockdown of HOTAIR inhibits tumour growth and blocks tumour invasion, several important observations with human specimens suggest a unique value of HOTAIR as a molecular prognostic marker of ESCC.

The incidence and mortality rate of EC is the highest in the Asian countries that stretch from Northern Iran through the central Asian republics to North-Central China, which is referred to as the 'EC belt'. Approximately $90 \%$ of the EC in these areas is SSC, which develops as a result of complex interactions between environmental, genetic, and epigenetic factors (Jemal et al, 2011); however, these interactions are poorly understood. Numerous epidemiological studies have revealed that environmental and dietary factors, such as smoking and smokeless tobacco consumption, betel quid chewing, alcohol intake, and micronutrient deficiency are associated with the aetiology of ESCC in high-risk areas (Lee et al, 2005). However, in certain high-risk areas, such as Linzhou, Henan, and China, these risk factors have a less important role (Tran et al, 2005). Studies have shown that the familial component may be more significant in high-incidence areas in northern China, suggesting that genetic susceptibility may have a pivotal role in oesophageal carcinogenesis (Zhang et al, 2000). As in other solid tumours, the inactivation of tumour suppressor genes (TSGs) and the activation of oncogenes occur during ESCC development and progression. Moreover, heritable polymorphisms in various carcinogen-metabolizing genes modulate the effect of environmental carcinogens and further increase the risk of ESCC (Malik et al, 2010). The interaction of tobaccorelated carcinogens and carcinogen-metabolizing genes such as GSTM1 and GSTT1 was found to modulate the effect of tobacco exposure and increase the susceptibility of developing ESCC (Jain et al, 2006).

Epigenetic modifications, which include modifications to DNA (that is, DNA methylation), modifications to histone proteins at specific amino-acid residues (for example, acetylation, methylation, and phosphorylation), and chromatin remodelling, have been thought to contribute to the regulation of gene expression. In addition to genetic factors, the epigenetic silencing of TSGs by aberrant promoter methylation has a significant role in the initiation and development of cancer, and the presence of methylated TSGs in tissue biopsies and body fluids can serve as a tumour biomarker (Robertson, 2005). Epigenetic events, such as

Figure 5. Gene expression profiling data and overall relation between differential methylation and expression. (A) Heat map of expression profiles for differentially expressed genes overlapped with cancer-associated genes set in the Molecular Signatures Database. The colour of the heat map represents the relative expression of each sample (red: overexpressed; green: underexpressed). Clustering is performed using an average clustering algorithm and a Euclidean distance dissimilarity metric of the normalised expression. (B-D) A selection of the top enriched GO biological process. Gene ontology molecular function, and Kyoto Encyclopedia of Genes and Genomes pathway for genes with a $P$-value $\leqslant 0.05$. The numbers for each pathway indicate the fold enrichment based on the Fisher exact test. (E) Total number of hypermethylated $\mathrm{CpG}$ sties in KYSE180 and KYSE180_HOTAIR cells over a basal $\beta$-value of 0.6. (F) Scatter plots of $\beta$-values show differentially methylated loci between KYSE180 cells vs KYSE180_HOTAIR cells as measured by Infinium HumanMethylation450K BeadArrays. The differential methylation cutoff (pink and blue dotted line) was estimated by controlling false discovery rate $<0.05$. The colour scale indicates the genes with corresponding levels of methylation (increases from blue to red). (G) Graphic showing the percentage of differentially methylated CpG sites in KYSE180_HOTAIR cells with respect to KYSE180 cells, percentage of hypermethylation and hypomethylation. $(\mathbf{H}) \mathrm{CpG}$ hypermethylation and hypomethylation events observed in KYSE180_HOTAIR cells in comparison with control KYSE180 cells according to functional genomic distribution (promoter, intergenic, 5'-UTR, 3'-UTR, gene body, and 1st exon). (I) CpG hypermethylation and hypomethylation events observed in KYSE180_HOTAIR cells in comparison with control KYSE180 cells according to chromosome location. (J) Overall relation between differential methylation and expression. Shown is a scatter plot of the relative levels (according to DiffScro in Infinium HumanMethylation450K BeadArrays) of methylation and fold change (according to log2 transformed data) in gene expression from microarray. Pink-coloured dots represent that a set of genes decrease methylation and increased expression, blue-coloured dots represent that a set of genes increased methylation and decreased expression in KYSE180_HOTAIR cells. (K) A summary of coordinate and independent regulation of genes by methylation changes in KYSE180_HOTAIR cells compared with KYSE180 cells. (L) Gene expression was determined by qRT-PCR in HOTAIR knockdown and HOTAIR overexpression cells, and relative gene expression determinations were made with the comparative delta-delta CT method $\left(2^{-\Delta \Delta C t}\right)$. The results are expressed as means $\pm s$. d. of three independent experiments. ${ }^{\star} P<0.05 ;{ }^{\star \star} P<0.01$; ${ }^{\star \star \star} P<0.001$. 
the aberrant DNA methylation of TSGs, which are involved in diverse cellular pathways including cell cycle regulation ( $p 16)$, apoptosis $(D A P K)$, DNA repair (BRCA1), and DNA protection (GSTP1), are considered to be important factors in the development and progression of ESCC (Li et al, 2011; Lima et al, 2011). In addition to protein-coding genes, ncRNAs are also regulated by epigenetic modifications and participate in the development and progression of EC. One lncRNA, AFAP1-AS1, was markedly hypomethylated and overexpressed in Barrett's oesophagus and EAC tissues and cells. The deregulated expression of AFAP-AS1 is involved in the development and progression of EAC, and AFAP-AS1 is a functional lncRNA in oesophageal carcinogenesis (Wu et al, 2013).

There is increasing evidence that lncRNA elements coordinate the molecular integration of the information flow that is required to deliver the right transcripts to the right location at the right time. Long ncRNAs were recently shown to control gene transcription via several different pathways: transcriptional gene silencing through the targeted recruitment of epigenetic silencing complexes to particular loci (Mercer and Mattick, 2013); posttranscriptional gene silencing; degradation of transcriptionally active mRNAs (Li et al, 2013b), which happens during RNA interference by siRNAs and microRNAs; and STAU-1-mediated RNA decay (Gong and Maquat, 2011). Notably, 20\% of the lncRNAs expressed in humans were bound by the components of PRC2 either alone or in combination with other chromatin remodelling complexes, such as those formed by CoREST and SMCX, and mediated the epigenetic modulation of chromatin states to regulate gene expression (Khalil et al, 2009). HOX transcript antisense RNA has been reported to physically associate with PRC2 and to epigenetically regulate multiple target genes. The upregulation of HOTAIR drives the development of the malignant phenotype in gastrointestinal stromal tumours and promotes cell invasion by altering the expression of HOTAIR target genes (Niinuma et al, 2012). Consistent with these reports, our data demonstrated that the depletion of HOTAIR induced a significant change in the gene expression profile of ESCC cells, suggesting that HOTAIR may regulate a spectrum of genes in addition to those previously reported. Pathway and GO analysis indicated that the differentially expressed gene sets were implicated in tumorigenesis and were significantly enriched in processes such as cell differentiation, development, cell death, adhesion, mobility, the cell cycle, and focal adhesion, providing important clues for understanding the molecular mechanism of ESCC pathogenesis. Among the processes and genes highlighted by this work, many have been previously characterised in ESCC pathogenesis, such as focal adhesion, the mitogen-activated protein kinase pathway, and p53. Developing a strategy to silence the expression of oncogenic lncRNAs, such as HOTAIR, will undoubtedly yield important clinical breakthroughs for cancer patients.

Epigenetic gene regulation is often achieved through histone modifications in which di- or trimethylation of histone $\mathrm{H} 3$ lysine 4 (H3K4 me2 or H3K4 me3) is enriched within active gene promoters. In addition, trimethylation of histone H3 lysine 27 (H3K27 me3) is a marker of gene silencing (Simon and Kingston, 2013). In normal fibroblasts, HOTAIR guides the PRC2 complex to the HOXD cluster and genes on other chromosomes where it transrepresses transcription (Rinn et al, 2007). The interaction of HOTAIR with PRC2 subunits and its subsequent regulation of transcription show that ncRNAs can target PRC2 to HOXD and other target genes. Although Hox ncRNAs are thought to be involved in cis regulation, HOTAIR depletion did not affect the genes in the HOXC cluster in cis. This posed a key question: can HOTAIR directly target PRC2 to another chromosome and, if so, how? To ascertain whether HOTAIR affects the DNA methylation pattern and participates in regulating the gene expression levels in ESCC cells, we compared the gene expression profiles with the results from the genome-wide DNA methylation analysis in KYSE180 and KYSE180_HOTAIR cells. These experiments revealed that only a small proportion of the methylation changes correlated with gene expression changes. Although this study only focused on a pair of cell lines, these findings support the importance of the role of HOTAIR in epigenetic regulation in ESCC. Further studies, including genome-wide histone modification analysis, may reveal as-yet unidentified roles for HOTAIR in the malignant progression of ESCC.

In summary, although our understanding of ESCC pathogenesis has improved through the identification of activating mutations in and amplification of oncogenes, including cyclin D1, EGFR, and $\mathrm{c}-\mathrm{Myc}$, and inactivating mutations in TSGs, such as p53 and p16 (Stoner and Gupta, 2001; Enzinger and Mayer, 2003; Kim et al, 2006), the mechanism of ESCC progression, including the role of cell proliferation, apoptosis resistance, invasion, metastasis, and angiogenesis, has not been elucidated. Our study provides key evidence to support the hypothesis that the overexpression of the oncogenic lncRNA HOTAIR, in addition to the deregulation of protein-coding genes, is clinically and functionally relevant in the progression of human ESCC. The elevated expression of HOTAIR has been associated with poor prognosis, which is probably because of the ability of HOTAIR to promote anti-apoptotic processes while inducing the invasive and metastatic behaviour of ESCC cells. Cumulatively, these findings indicate that HOTAIR has a vital role in the development and progression of ESCC. Understanding the precise role of HOTAIR in the pathogenesis of ESCC will increase our understanding of the biological basis of cancer progression and may lead to the development of a novel diagnostic marker and therapeutic strategy for ESCC.

\section{ACKNOWLEDGEMENTS}

We thank anonymous reviewers for helpful comments on the manuscript and all the patients for consenting to provide tissue samples. This research was supported by the grants from the National Natural Science Foundation of China (number 31201033 to XLL, number 31270820 and number 81230061 to $\mathrm{WDH}$, and number 81121004 to XBF) and was partially supported by a grant from the National Basic Science and Development Programme of China (number 2012CB518103 to WDH and number 2010CB912802 to MZG).

\section{CONFLICT OF INTEREST}

The authors declare no conflict of interest.

AUTHOR CONTRIBUTIONS

XLL planned and performed experiments, performed data analysis and wrote the manuscript; ZQW, QM, and XL performed experiments and collected and analysed data; MZG provided histopathology expertise; WDH and XBF conceived the study design and reviewed the manuscript; XLL, ZQW, QM, and WDH were involved in writing the paper and had final approval of the submitted and published versions.

\section{REFERENCES}

Allum WH, Stenning SP, Bancewicz J, Clark PI, Langley RE (2009) Long-term results of a randomized trial of surgery with or without preoperative chemotherapy in esophageal cancer. J Clin Oncol 27(30): 5062-5067. 
Beissbarth T, Speed TP (2004) GOstat: find statistically overrepresented Gene Ontologies within a group of genes. Bioinformatics 20(9): 1464-1465.

Berezikov E, Plasterk RH (2005) Camels and zebrafish, viruses and cancer: a microRNA update. Hum Mol Genet 14 Spec No. 2: R183-R190.

Borchert GM, Lanier W, Davidson BL (2006) RNA polymerase III transcribes human microRNAs. Nat Struct Mol Biol 13(12): 1097-1101.

Carthew RW, Sontheimer EJ (2009) Origins and mechanisms of miRNAs and siRNAs. Cell 136(4): 642-655.

Enzinger PC, Mayer RJ (2003) Esophageal cancer. N Eng J Med 349(23): 2241-2252.

Esteller M (2008) Epigenetics in cancer. NEng J Med 358(11): 1148-1159.

Farazi TA, Spitzer JI, Morozov P, Tuschl T (2011) miRNAs in human cancer. I Pathol 223(2): 102-115.

Geng YJ, Xie SL, Li Q, Ma J, Wang GY (2011) Large intervening non-coding RNA HOTAIR is associated with hepatocellular carcinoma progression. J Int Med Res 39(6): 2119-2128.

Gong C, Maquat LE (2011) lncRNAs transactivate STAU1-mediated mRNA decay by duplexing with 3' UTRs via Alu elements. Nature 470(7333): 284-288.

Gupta RA, Shah N, Wang KC, Kim J, Horlings HM, Wong DJ, Tsai MC, Hung T, Argani P, Rinn JL, Wang Y, Brzoska P, Kong B, Li R, West RB, van de Vijver MJ, Sukumar S, Chang HY (2010) Long non-coding RNA HOTAIR reprograms chromatin state to promote cancer metastasis. Nature 464(7291): 1071-1076.

Guttman M, Donaghey J, Carey BW, Garber M, Grenier JK, Munson G, Young G, Lucas AB, Ach R, Bruhn L, Yang X, Amit I, Meissner A, Regev A, Rinn JL, Root DE, Lander ES (2011) lincRNAs act in the circuitry controlling pluripotency and differentiation. Nature 477(7364): 295-300.

Hildebrandt MA, Yang H, Hung MC, Izzo JG, Huang M, Lin J, Ajani JA, Wu X (2009) Genetic variations in the PI3K/PTEN/AKT/mTOR pathway are associated with clinical outcomes in esophageal cancer patients treated with chemoradiotherapy. J Clin Oncol 27(6): 857-871.

Ishibashi M, Kogo R, Shibata K, Sawada G, Takahashi Y, Kurashige J, Akiyoshi S, Sasaki S, Iwaya T, Sudo T, Sugimachi K, Mimori K, Wakabayashi G, Mori M (2013) Clinical significance of the expression of long non-coding RNA HOTAIR in primary hepatocellular carcinoma. Oncol Rep 29(3): 946-950.

Jain M, Kumar S, Rastogi N, Lal P, Ghoshal UC, Tiwari A, Pant MC, Baiq MQ, Mittal B (2006) GSTT1, GSTM1 and GSTP1 genetic polymorphisms and interaction with tobacco, alcohol and occupational exposure in esophageal cancer patients from North India. Cancer Lett 242(1): 60-67.

Jemal A, Bray F, Center MM, Ferlay J, Ward E, Forman D (2011) Global cancer statistics. CA Cancer J Clin 61(2): 69-90.

Jones PA, Baylin SB (2002) The fundamental role of epigenetic events in cancer. Nat Rev Genet 3(6): 415-428.

Khalil AM, Guttman M, Huarte M, Garber M, Raj A, Rivea Morales D, Thomas K, Presser A, Bernstein BE, van Oudenaarden A, Regev A, Lander ES, Rinn JL (2009) Many human large intergenic noncoding RNAs associate with chromatin-modifying complexes and affect gene expression. Proc Natl Acad Sci USA 106(28): 11667-11672.

Kim K, Jutooru I, Chadalapaka G, Johnson G, Frank J, Burghardt R, Kim S, Safe S (2013) HOTAIR is a negative prognostic factor and exhibits prooncogenic activity in pancreatic cancer. Oncogene 32(13): 1616-1625.

Kim SH, Nakagawa H, Navaraj A, Naomoto Y, Klein-Szanto AJ, Rustgi AK, El-Deiry WS (2006) Tumorigenic conversion of primary human esophageal epithelial cells using oncogene combinations in the absence of exogenous Ras. Cancer Res 66(21): 10415-10424.

Kogo R, Shimamura T, Mimori K, Kawahara K, Imoto S, Sudo T, Tanaka F, Shibata K, Suzuki A, Komune S, Miyano S, Mori M (2011) Long noncoding RNA HOTAIR regulates polycomb-dependent chromatin modification and is associated with poor prognosis in colorectal cancers. Cancer Res 71(20): 6320-6326.

Lee CH, Lee JM, Wu DC, Hsu HK, Kao EL, Huang HL, Wang TN, Huang MC, Wu MT (2005) Independent and combined effects of alcohol intake, tobacco smoking and betel quid chewing on the risk of esophageal cancer in Taiwan. Int J Cancer 113(3): 475-482.

Li B, Wang B, Niu LJ, Jiang L, Qiu CC (2011) Hypermethylation of multiple tumor-related genes associated with DNMT3b up-regulation served as a biomarker for early diagnosis of esophageal squamous cell carcinoma. Epigenetics 6(3): 307-316.

Li DD, Feng JP, Wu TY, Wang YD, Sun YN, Ren JY, Liu M (2013a) Long intergenic noncoding RNA HOTAIR is overexpressed and regulates PTEN methylation in laryngeal squamous cell carcinoma. Am J Pathol 182(1): 64-70.

Li X, Wu Z, Fu X, Han W (2013b) Long noncoding RNAs: insights from biological features and functions to diseases. Med Res Rev 33(3): 517-553.

Lima SC, Hernandez-Vargas H, Simao T, Durand G, Kruel CD, Le Calvez-Kelm F, Ribeiro Pinto LF, Herceg Z (2011) Identification of a DNA methylome signature of esophageal squamous cell carcinoma and potential epigenetic biomarkers. Epigenetics 6(10): 1217-1227.

Lin DC, Du XL, Wang MR (2009) Protein alterations in ESCC and clinical implications: a review. Dis Esophagus 22(1): 9-20.

Lu J, Getz G, Miska EA, Alvarez-Saavedra E, Lamb J, Peck D, Sweet-Cordero A, Ebert BL, Mak RH, Ferrando AA, Downing JR, Jacks T, Horvitz HR, Golub TR (2005) MicroRNA expression profiles classify human cancers. Nature 435(7043): 834-838.

Malik MA, Upadhyay R, Mittal RD, Zargar SA, Mittal B (2010) Association of xenobiotic metabolizing enzymes genetic polymorphisms with esophageal cancer in Kashmir Valley and influence of environmental factors. Nutr Cancer 62(6): 734-742.

Mattick JS (2004) RNA regulation: a new genetics? Nat Rev Genet 5(4): 316-323.

Medical Research Council Oesophageal Cancer Working G (2002) Surgical resection with or without preoperative chemotherapy in oesophageal cancer: a randomised controlled trial. Lancet 359(9319): 1727-1733.

Mercer TR, Dinger ME, Mattick JS (2009) Long non-coding RNAs: insights into functions. Nat Rev Genet 10(3): 155-159.

Mercer TR, Dinger ME, Sunkin SM, Mehler MF, Mattick JS (2008) Specific expression of long noncoding RNAs in the mouse brain. Proc Natl Acad Sci USA 105(2): 716-721.

Mercer TR, Mattick JS (2013) Structure and function of long noncoding RNAs in epigenetic regulation. Nat Struct Mol Biol 20(3): 300-307.

Nelson KM, Weiss GJ (2008) MicroRNAs and cancer: past, present, and potential future. Mol Cancer Ther 7(12): 3655-3660.

Nie Y, Liu X, Qu SH, Song EW, Zou H, Gong C (2013) Long noncoding RNA HOTAIR is an independent prognostic marker for nasopharyngeal carcinoma progression and survival. Cancer Sci 104(4): 458-464.

Niinuma T, Suzuki H, Nojima M, Nosho K, Yamamoto H, Takamaru H, Yamamoto E, Maruyama R, Nobuoka T, Miyazaki Y, Nishida T, Bamba T, Kanda T, Ajioka Y, Taguchi T, Okahara S, Takahashi H, Nishida Y, Hosokawa M, Hasegawa T, Tokino T, Hirata K, Imai K, Toyota M, Shinomura Y (2012) Upregulation of miR-196a and HOTAIR drive malignant character in gastrointestinal stromal tumors. Cancer Res 72(5): $1126-1136$.

Pillai RS, Bhattacharyya SN, Filipowicz W (2007) Repression of protein synthesis by miRNAs: how many mechanisms? Trends Cell Biol 17(3): $118-126$.

Pohl H, Welch HG (2005) The role of overdiagnosis and reclassification in the marked increase of esophageal adenocarcinoma incidence. J Natl Cancer Inst 97(2): 142-146.

Ponjavic J, Ponting CP, Lunter G (2007) Functionality or transcriptional noise? Evidence for selection within long noncoding RNAs. Genome Res 17(5): 556-565.

Ponting CP, Oliver PL, Reik W (2009) Evolution and functions of long noncoding RNAs. Cell 136(4): 629-641.

Prasanth KV, Spector DL (2007) Eukaryotic regulatory RNAs: an answer to the 'genome complexity' conundrum. Genes Dev 21(1): 11-42.

Rinn JL, Kertesz M, Wang JK, Squazzo SL, Xu X, Brugmann SA, Goodnough LH, Helms JA, Farnham PJ, Segal E, Chang HY (2007) Functional demarcation of active and silent chromatin domains in human HOX loci by noncoding RNAs. Cell 129(7): 1311-1323.

Rizk NP, Ishwaran H, Rice TW, Chen LQ, Schipper PH, Kesler KA, Law S, Lerut TE, Reed CE, Salo JA, Scott WJ, Hofstetter WL, Watson TJ, Allen MS, Rusch VW, Blackstone EH (2010) Optimum lymphadenectomy for esophageal cancer. Annals Surg 251(1): 46-50.

Robertson KD (2005) DNA methylation and human disease. Nat Rev Genet 6(8): 597-610.

Simon JA, Kingston RE (2013) Occupying chromatin: polycomb mechanisms for getting to genomic targets, stopping transcriptional traffic, and staying put. Mol Cell 49(5): 808-824.

Stoner GD, Gupta A (2001) Etiology and chemoprevention of esophageal squamous cell carcinoma. Carcinogenesis 22(11): 1737-1746. 
Tran GD, Sun XD, Abnet CC, Fan JH, Dawsey SM, Dong ZW, Mark SD, Qiao YL, Taylor PR (2005) Prospective study of risk factors for esophageal and gastric cancers in the Linxian general population trial cohort in China. Int J Cancer 113(3): 456-463.

Tsai MC, Manor O, Wan Y, Mosammaparast N, Wang JK, Lan F, Shi Y, Segal E, Chang HY (2010) Long noncoding RNA as modular scaffold of histone modification complexes. Science 329(5992): 689-693.

Wapinski O, Chang HY (2011) Long noncoding RNAs and human disease. Trends Cell Biol 21(6): 354-361.

Wu W, Bhagat TD, Yang X, Song JH, Cheng Y, Agarwal R, Abraham JM, Ibrahim S, Bartenstein M, Hussain Z, Suzuki M, Yu Y, Chen W, Eng C, Greally J, Verma A, Meltzer SJ (2013) Hypomethylation of noncoding DNA regions and overexpression of the long noncoding RNA, AFAP1AS1, in Barrett's esophagus and esophageal adenocarcinoma. Gastroenterology 144(5): 956-966e4.

Yang Z, Zhou L, Wu LM, Lai MC, Xie HY, Zhang F, Zheng SS (2011) Overexpression of long non-coding RNA HOTAIR predicts tumor recurrence in hepatocellular carcinoma patients following liver transplantation. Annals Surg Oncol 18(5): 1243-1250.
Zeller C, Dai W, Steele NL, Siddiq A, Walley AJ, Wilhelm-Benartzi CS, Rizzo S, van der Zee A, Plumb JA, Brown R (2012) Candidate DNA methylation drivers of acquired cisplatin resistance in ovarian cancer identified by methylome and expression profiling. Oncogene 31(42): 4567-4576.

Zhang W, Bailey-Wilson JE, Li W, Wang X, Zhang C, Mao X, Liu Z, Zhou C, Wu M (2000) Segregation analysis of esophageal cancer in a moderately high-incidence area of northern China. Am J Hum Genet 67(1): 110-119.

Zieve GW, Turnbull D, Mullins JM, McIntosh JR (1980) Production of large numbers of mitotic mammalian cells by use of the reversible microtubule inhibitor nocodazole. Nocodazole accumulated mitotic cells. Exp Cell Res 126(2): 397-405.

This work is published under the standard license to publish agreement. After 12 months the work will become freely available and the license terms will switch to a Creative Commons AttributionNonCommercial-Share Alike 3.0 Unported License.

Supplementary Information accompanies this paper on British Journal of Cancer website (http://www.nature.com/bjc) 